\title{
A Brief Review of Independent, Dependent and One Sample t-test
}

\section{Banda Gerald}

Department of Mathematics and Statistics, Mukuba University, Itimpi Campus, Kitwe, Zambia

Email address:

gerald1banda@gmail.com

\section{To cite this article:}

Banda Gerald. A Brief Review of Independent, Dependent and One Sample t-test. International Journal of Applied Mathematics and Theoretical Physics. Vol. 4, No. 2, 2018, pp. 50-54. doi: 10.11648/j.ijamtp.20180402.13

Received: June 27, 2018; Accepted: July 17, 2018; Published: August 14, 2018

\begin{abstract}
In statistics it is important to know the method of analysing data. The study looked at parametric tests with a special focus on the three t-tests. In this paper, the researcher provide a brief review of commonly used three t-tests namely an independent sample t-test, dependent sample-test and one sample t-test. The researcher has presented the formulae for calculating an independent sample t-test, dependent sample-test and one sample t-test. Procedures have also been written in this article on how to conduct the three t-tests in SPSS. The article has also looked at the formulae for calculating the effect size and its important when conducting a study.
\end{abstract}

Keywords: Independent Sample t-test, Dependent Sample t-test, One Sample t-test, Effect Size

\section{Introduction}

The main purpose of conducting a quantitative research at any level of education is to find the solution to the problem. In order to avoid statistical errors, appropriate methods of analysing data should be applied. There are two sets of statistical tests for comparing means and these are: Parametric tests and non-parametric tests. Parametric tests work with normally distributed scale data while nonparametric tests are used with not normally distributed scale data and with ordinal data. Within parametric and nonparametric tests, some work with just two means while others work with more than two means. The t-test is an example of the parametric test that works on normally distributed scale data and compares two means. According to Pallant (2007), t-tests are used when you have two groups or two sets of data. For example, t-test can be used by the researcher to find out the impact of teaching methods on the performance of students in Mathematics. According to McMillan and Schumacher (2010), t-test refers to an inferential statistical procedure for determining the probability level of rejecting the null hypothesis that two means are the same. T-test assumes that the population from which the sample is drawn is normal and sample observations are independent. T-test can only be applied if the sample size is small $(n \leq 30)$. T- test is applied to test the significance of the mean of the random samples and the difference between the means of two samples. There are three types of t-tests and these are: Independent sample t-test, dependent sample t-test and one sample t-test. One sample t-test compares the mean of a sample to a predefined value. Dependent (related, within subject or paired) sample t-test compares the means of two conditions in which the same (or closely matched) participants participated in the study. Independent (unrelated) sample t-test compares the means of two groups of participants.

In statistics it is necessary to test for normality in order to decide whether to use parametric tests or non-parametric tests. The entire statistical framework is grounded on the assumption of normality and if this assumption is violated, then inference breaks down. For this reason it is essential to check or test the assumption of normality before any statistical analysis of data. The assumption of normality should be checked before analysing data when comparing the means of two or more groups because the validity of the results depend on the test of normality. In order to test for normality, we need to test whether a sample of observations comes from a normal distribution (Henry, 2002). According to Pallant (2007), the assumption of normality can be tested using either Kolmogorov-Smirnov test or Shapiro-Wilks test. Kolmogorov-Smirnov test works best if the sample size is 
more than fifty (50) while Shapiro-Wilks test is used to test for normality of the data if the sample size is less than fifty (50). It is also worthy of knowing whether the difference between two groups occurred by chance or not. The objective of this article is to review the independent sample t-test, dependent sample t-test and one sample t-test. The focus on independent sample t-test, dependent sample t-test and one sample t-test is on how you can calculate both using the formulae and using the Statistical Package for Social Science (SPSS). The paper has also looked at how to calculate effect size for both the independent sample t-test and dependent sample t-test. It is hoped that one who follows this article may be able to distinguish between the independent sample ttest and dependent sample t-test.

\subsection{Independent Sample t-test}

Independent samples $t$-test is used to compare two groups whose means are not dependent on one another (University of Arizona Military Reach, 2009). Two samples are independent if the sample values selected from one population are not related or somehow paired or matched with the sample values selected from the other population. An independent sample t-test tells the researcher whether there is a statistically significant difference in the mean scores for the two groups or not. In statistical terms it means that the researcher is testing the probability that the two sets of data came from the same population. In other words, an independent sample is the sample in which the participants in each group are independent from each other. A common example of independent groups might be comparisons between students taught using Collaboration Learning Approach and the other group taught using Conventional Learning Approach. In this example the factor that differentiates the two groups is the teaching method. Teaching method does not indicate that the scores from group taught using Collaboration Learning Approach will be dependent on scores from the other group taught using Conventional Learning Approach. An independent sample ttest can only be used if the sample is coming from the normal population.

\subsection{Procedures When Conducting an Independent Sample t-test}

The first step is to state the null hypothesis and alternative hypothesis. According to Marilyn and Theresa (2003), the null hypothesis is a statement about a parameter of the population (s) and is labelled $H_{0}$. The alternative hypothesis is a statement about a parameter of the population (s) that is opposite to the null hypothesis and is labelled $H_{1}$.

Hypothesis

$H_{0}: \bar{X}_{1}=\bar{X}_{2}$ (there is no statistically signific9ant difference between the two sample means).

$H_{1}: \bar{X}_{1} \neq \bar{X}_{2}$ (there is a statistically significant difference between the two sample means).

The second step is to state the level of significance ( $\alpha$-level). The significance level $\alpha$ is the maximum probability tolerated for rejecting a null hypothesis (Marilyn and Theresa 2003). The third level is to compute the test statistics. According to Marilyn and Theresa (2003), a test statistic is a number that captures information in the sample data. It is used to decide between the null hypothesis and alternative hypothesis. The fourth step is to compare the calculated value and the value from the table. The last step is to summarise and make conclusion based on the calculated value and the table value. Independent sample t-test can be calculated using the formula or Statistical Package for Social Science (SPSS). The following is the formula that can be used to calculate an independent sample t-test.

$$
t=\frac{\bar{X}_{1}-\bar{X}_{2}}{\sqrt{\frac{\sum_{i=1}^{n} x_{i 1}^{2}-\frac{\left(\sum_{i=1}^{n} x_{i 1}\right)^{2}}{n_{1}}+\sum_{i=1}^{n} x_{i 2}^{2}-\frac{\left(\sum_{i=1}^{n} x_{i 2}\right)^{2}}{n_{2}}}{n_{1} n_{2}\left(1-\frac{2}{n_{1}+n_{2}}\right)}}}
$$

From the formula above,

$n_{1}$ is the sample size of the first group and $n_{2}$ is the sample size of the second group.

$\bar{X}_{1}$ is the sample mean of the first group and $\bar{X}_{2}$ is the sample mean of the second group. The sample mean of the first group can be calculated using the formula

$$
\bar{X}_{1}=\frac{\sum_{i=1}^{n} x_{i 1}}{n_{1}}
$$

While the sample mean of the second group can be calculated using the formula

$$
\bar{X}_{2}=\frac{\sum_{i=1}^{n} x_{i 2}}{n_{2}}
$$

$\sum_{i=1}^{n} x_{i 1}$ is the sum of scores of the first group and $\sum_{i=1}^{n} x_{i 2}$ is the sum of scores of the second group.

$\sum_{i=1}^{n} x_{i 1}^{2}$ is the sum of squared scores of the first group and $\sum_{i=1}^{n} x_{i 2}^{2}$ is the sum of squared scores of the second group.

The following are the procedures to follow when calculating an independent sample t-test using SPSS:

The first thing to do under variable view is to write group (when conducting research) in first column under the first row and then code the two groups under values in the same first row. The second thing to do under variable view is to write scores in first column under the second row. Under data view: In data view we go to analyse and then click compare means. From compare means we go to independent sample ttest. Under independent sample t-test input, scores (dependent variable) is the test variable and independent variable (categorical variable) is the grouping variable. Under independent sample t-test input, move the dependent variable into the test variable and independent variable (categorical variable) into the grouping variable. Click on define groups and write the groups the way they have been coded in variable view. Then click on continue and finally click on ok.

\subsection{Dependent Sample t-test}

Dependent sample t-test, sometimes called the paired 
sample t-test is used when the observations on the two populations of interest are collected in pairs (Douglas and George, 2003). Two samples are dependent (or consist of matched pairs) if the members of one sample can be used to determine the members of the other sample. The words like dependent, repeated, before and after, matched pairs, paired and so on are hints for dependent samples. According to University of Arizona Military Reach (2009), dependent Samples $t$-test is used to compare two groups of scores and their means in which the participants in one group are somehow meaningfully related to the participants in the other group. One common example of such a relation is in a pretest post-test research design. Participants at the pre-test are the same participants at the post-test and the scores between pre- and post-test are meaningfully related. What it means is that the scores between pre- and post-test are dependent on each other. University of Arizona Military Reach (2009) further explained that other forms of dependency may also exist such as scores between parents and their children or between dating couples. For example, the researcher may decide to conduct a research on the impact of Problem Solving Approach on the performance of students using one group. Before teaching students using Problem Solving Approach, pre-test should be given. After teaching students using Problem Solving Approach, post-test should be given in order to compare the scores for pre-test and post-test. In this case scores from two groups are likely to be related. Conventional Learning Approach would initially be measured to obtain pre-test scores. Next, the intervention (Problem Solving Approach) would be administered followed by a second measurement (post-test). In order to determine if there was an increase in performance as a result of the intervention, the researcher would use a dependent samples $t$ test that accounts for the meaningful relation between participants at the pre-test and post-test. Just like an independent sample t-test, dependent sample t-test can only be used if the assumption of normality is conducted and the results shows that the sample is coming from the normal population.

Procedures when conducting dependent sample t-test

Procedures used to conduct dependent sample t-test are the same as the procedures when conducting an independent sample t-test. Dependent sample t-test can be calculated using the formula or Statistical Package for Social Science (SPSS). The following is the formula that can be used to calculate dependent sample t-test.

$$
t=\frac{1}{\sqrt{\frac{\sum_{i=1}^{n}\left(\frac{d_{i}}{\bar{d}}-1\right)^{2}}{n(n-1)}}}
$$

Where

$n$ is the sample size of the study.

$d_{i}$ are the differences between the first result and the second result of each individual participant in the study. $d_{i}$ is calculated using the formula $d_{i}=x_{i 1}-x_{i 2}$ where $i=1,2,3, \ldots, n$ (each participant in the study)

$x_{i 1}$ : First results of each participant in the study.

$x_{i 2}$ : Second results of each participant in the study.

$\bar{d}$ is the sample mean of the sum of differences between the first results and the second results of participants in the study.

$$
\bar{d}=\frac{\sum_{i=1}^{n} d_{i}}{n}
$$

Or

$$
\bar{d}=\bar{X}_{1}-\bar{X}_{2}
$$

$\bar{X}_{1}$ is the sample mean of the scores from the first results of the study and $\bar{X}_{2}$ is the sample mean of the scores from the second results of the study. The sample mean of the first results can be calculated using the formula

$$
\bar{X}_{1}=\frac{\sum_{i=1}^{n} x_{i 1}}{n}
$$

While the sample mean of the second results can be calculated using the formula

$$
\bar{X}_{2}=\frac{\sum_{i=1}^{n} x_{i 2}}{n}
$$

$\sum_{i=1}^{n}\left(d_{i}-\bar{d}\right)^{2}$ is the squared sum of scores of the differences between the first result and the second result of each individual participant in the study. $d_{i}-\bar{d}$ is calculated by subtracting the sample mean of the sum of differences between the first results and the second results from the differences between the first result and the second result of each individual participant in the study.

Dependent sample t-test can also be calculated using SPSS. The following are the procedures to follow when calculating dependent sample t-test using SPSS:

The first thing to do under variable view is to write pre-test (when conducting research) in first column under the first row and then write post-test in first column under the second row. Under data view: In data view we go to analyse and then click compare means. From compare means we go to paired sample t-test. Under paired sample t-test input, move pre-test scores into variable 1 and post-test scores into variable 2 and finally click on ok.

\subsection{One Sample t-test}

The one-sample t-test is used to compare a sample mean to a specific value. A researcher can use one-sample t-test to compare the mean of a sample with a hypothesized population mean to see if the sample is significantly different. One-sample t-test is used for example, to compare the sample mean and the sample midpoint of the test variable and also to determine whether a sample of observation could have been generated by a process with a specific mean. One sample t-test assumes that the dependent variable is normally distributed within the population and data is independent 
(scores of one participant are not dependent on scores of the others; participants are independent of one another). Before using one sample t-test, test of normality should be conducted.

Procedures when conducting one sample t-test

Procedures used to conduct one sample t-test are the same as the procedures when conducting an independent sample ttest and dependent sample t-test. One sample t-test can be calculated using the formula or Statistical Package for Social Science (SPSS). The following is the formula that can be used to calculate dependent sample t-test.

$$
\begin{gathered}
t=\frac{\bar{X}-\mu}{\frac{\hat{\sigma}}{\sqrt{n}}} \\
\hat{\sigma}_{\bar{X}}=\frac{\hat{\sigma}}{\sqrt{n}}
\end{gathered}
$$

Where

$n$ is the sample size of the study.

$\hat{\sigma}_{\bar{X}}$ is the estimated population standard deviation of the mean.

$\bar{X}$ is the sample mean of the scores and can be calculated using the formula

$$
\bar{X}=\frac{\sum_{i=1}^{n} x_{i}}{n}
$$

$\mu$ is the population mean or hypothesized value.

$\sum_{i=1}^{n}\left(x_{i}-\bar{X}\right)^{2}$ is the squared sum of scores of the differences between the scores and the sample mean.

The following are the procedures to follow when calculating one sample t-test using SPSS:

The first thing to do under variable view is to write the variable being tested in first column under the first row. Under data view: In data view we go to analyse and then click compare means. From compare means we go to one sample t-test. Under one sample t-test input, move the scores being tested into test variable (s) box. Write the test value (population mean) into the box test value and finally click on ok.

\section{Effect Size}

Both independent sample t-test and dependent sample ttest will give the research an indication of whether the difference between the two groups is statistically significant (that is not likely to have occurred by chance). According to Pallant (2007), there is more to research when results are significant than just obtaining statistical significance. Effect size explains the degree to which the two variables are associated with one another. With large samples, even very small differences between groups can become statistically significant. This does not mean that the difference has any practical or theoretical significance (Pallant, 2007). Effect size (strength of the association) is used to assess the importance of the findings of the study. According to Pallant (2007), effect size is the statistics that indicates the relative magnitude of the differences between means or the amount of total variance in the dependent variable that is predictable from the knowledge of the levels of the levels of the independent variable. The calculated value of effect size provide an indication of the magnitude of the difference between the two groups. According to Andy (2009), effect sizes are useful because they provide an objective measure of the importance of an effect.

\subsection{Effect Size of an Independent Sample t-test}

An effect size is simply an objective and (usually) standardized measure of the magnitude of observed effect (Andy, 2009). Many measures of effect size have been proposed, the most common of which are Cohen's $d$, Pearson's correlation coefficient $r$ and the odds ratio. Correlation coefficient is used to measure the strength of relationship between two variables. However, correlation coefficient is also a very versatile measure of the strength of an experimental effect. From the researcher's point of view, correlation coefficient of 0 means there is no effect, and a value of 1 means that there is a perfect effect. The following is the formula used to calculate the effect size;

$$
r=\sqrt{\frac{t^{2}}{t^{2}+n_{1}+n_{2}-2}}
$$

Where

$t$ is the calculated value of the independent sample t-test $n$ is the sample size

$r=0.1$ (Small effect)

$r=0.3$ (Medium effect)

$r=0.5$ (Large effect)

\subsection{Effect Size of Dependent Sample t-test}

According to Pallant (2007), the most common used method for calculating effect size is the Eta Squared. The formula for simplified Eta Squared is given by

$$
\text { Eta Squared }=\frac{1}{1+\frac{n-1}{t^{2}}}
$$

Where

$t$ is the calculated value of the dependent sample t-test $\mathrm{n}$ is the sample size

The calculated value of the Eta Squared should be between 0 and 1 .

0.01 Small effect

0.06 Moderate effect

0.14 Large effect

\section{Conclusion}

It is important to know the type of test to use when analysing data. Otherwise we might draw wrong conclusions by committing either type I or type II error. Normality test should be conducted before using either independent sample t-test, dependent sample t-test or one sample t-test. All the 
three t-tests can be calculated using either Statistical Package for Social Science (SPSS) or by using formula. The article has provided general definitions of all the three t-tests and when to apply them when conducting a study. This article has also highlighted the importance of conducting the effect size when conducting a study.

\section{References}

[1] Andy (2009). Discovering Statistics using SPSS $3^{\text {rd }}$ Edition. SAGE Publication Ltd.

[2] Bester, G., Smit, M. E. and Swanepoel, C. H (2011). Tutorial letter 102 / 2011, Workbook for Research, University of South Africa.

[3] Daniel. M (2004). Doing Quantitative Research in Education with SPSS: New Delhi, India.

[4] Douglas C. M and George C. R (2003). Applied Statistics and Probability for Engineers $3^{\text {rd }}$ Edition. John Wiley \& Sons, Inc.

[5] George, A. M et al (2011). IBM SPSS for Introductory Statistics use and Interpretation Fourth Edition. Routledge Taylor \& Francis Group 270 Madison Avenue. New York, NY 10016.

[6] Kothari, C. R. (2004). Research Methodology: Methods and Techniques. Jaipur: NEW AGE.

[7] Marilyn, K. P and Theresa, M. S (2003). Elementary Statistics.
John Wiley and Sons, Inc. USA.

[8] Mario, F. T (2012). Elementary Statistics $11^{\mathrm{TH}}$ Edition. Pearson Education Inc. Boston, USA.

[9] McMillan, J and Schumacher, S (2010). Research in Education. Evidence base Inquiry. 7th Edition, International Edition Boston: Pearson Education Inc.

[10] Morten, W. F (2012). T-tests, Non-Parametric Tests, and Large Studies-A Paradox of Statistical Practice. BMC Medical Research Methodology.

[11] Nicholas, W (2011). Research Methods: The Basics. London: Routledge.

[12] Pallant, J. (2007). SPSS Survival Manual: A Step by Step to Data Analysis Using SPSS for Windows (Version 15). Sydney: Allen and Unwin.

[13] Pandey, R. M (2015). Commonly Used t-tests in Medical Research. Journal of the Practice of Cardiovascular Sciences. Volume 1. Issue 2.

[14] University of Arizona Military Reach (2009). Understanding t-tests.

[15] Tae, K. K (2015). T Test as a Parametric Statistics. Korean Journal of Anesthesiology. Korean J Anesthesiol. 2015 Dec; 68(6): 540-546.

[16] Younis, S (2015). The Bread and Butter of Statistical Analysis 't-tests': Uses and Misuses. Pakistan Journal of Medical Sciences. Pak J Med Sci.2015 Nov-Dec: 31(6):1558-1559. 